\title{
Subjective swallowing symptoms and related risk factors in COPD
}

\author{
Margareta Gonzalez Lindh (10 ${ }^{1,2}$, Andrei Malinovschi ${ }^{3}$, Eva Brandén ${ }^{4,1}$, \\ Christer Janson $\mathbb{1}^{5}$, Björn Ställberg ${ }^{6}$, Kristina Bröms $\mathbb{1}^{6,1}$ \\ Monica Blom Johansson ${ }^{2}$, Karin Lisspers ${ }^{6}$ and Hirsh Koyi ${ }^{4,1}$
}

Affiliations: ${ }^{1}$ Centre for Research and Development, Uppsala University/Region Gävleborg, Gävle, Sweden. ${ }^{2}$ Dept of Neuroscience, Uppsala University, Uppsala, Sweden. ${ }^{3}$ Dept of Medical Sciences, Clinical Physiology, Uppsala University, Uppsala, Sweden. ${ }^{4}$ Respir. Med. Unit, Dept Med. Solna and CMM, Karolinska Institutet and Karolinska University Hospital Solna, Solna, Sweden. ${ }^{5}$ Dept of Medical Sciences, Respiratory, Allergy and Sleep Research, Uppsala University, Uppsala, Sweden. ${ }^{6}$ Dept of Public Health and Caring Sciences, Family Medicine and Preventive Medicine, Uppsala University, Uppsala, Sweden.

Correspondence: Margareta Gonzalez Lindh, Centre for Research and Development, Uppsala University/ Region Gävleborg, CFUG Gävle Hospital 80188, Gävle, Sweden. E-mail: margareta.gonzalez.linda regiongavleborg.se

\section{ABSTRACT}

Objectives: This study aimed to investigate the prevalence of subjective (i.e. self-reported) swallowing symptoms in a large cohort of patients with stable chronic obstructive pulmonary disease (COPD) and to identify potential related risk factors.

Methods: A total of 571 patients with COPD, investigated in a stable phase, participated in this multicentre study (335 females, 236 males; mean age: 68.6 years (SD 7.7)). Data were derived from spirometry, a questionnaire and a 30-metre walking test.

Results: In total, 33\% $(\mathrm{n}=186)$ patients reported at least some degree of swallowing problem. The most frequently reported symptom was food lodging in the throat (23\%). A significant relationship was found between swallowing symptoms and dyspnoea, assessed as modified Medical Research Council (mMRC) $\geqslant 2$ compared with $<2(46 \%$ versus $22 \% ; \mathrm{p}<0.001)$ and health-related quality of life, assessed as the COPD Assessment Test $(\mathrm{CAT}) \geqslant 10$ (40\% versus 19\%; $\mathrm{p}<0.001)$. Swallowing problems were also related to lower physical capacity $(\mathrm{p}=0.02)$ but not to lung function $(\mathrm{p}>0.28)$.

Conclusion: Subjective swallowing symptoms seem to be a common problem in patients with stable COPD. This problem is seen in all stages of the disease, but is more common in symptomatic patients and in patients with lower physical capacity.

@ERSpublications

Patients with \#COPD can experience problems swallowing food and liquid efficiently and safely, according to new research from Margareta Gonzalez Lindh and colleagues at @UU_University http://bit.ly/2Kmxyqx

Cite this article as: Gonzalez Lindh M, Malinovschi A, Brandén E, et al. Subjective swallowing symptoms and related risk factors in COPD. ERJ Open Res 2019; 5: 00081-2019 [https://doi.org/ 10.1183/23120541.00081-2019]. 


\section{Introduction}

Chronic obstructive pulmonary disease (COPD) is a lung disease characterised by chronic airflow obstruction that interferes with normal breathing and is not fully reversible [1]. The prevalence in Sweden is approximately $10 \%$ in the adult population [2] which is in agreement with global reports [3]. There is presently no cure for COPD and it is the only endemic disease in Sweden for which mortality is increasing [4]. The number of patients with COPD is also increasing worldwide and it is the third most common cause of death globally [5]; thus its impact both from an epidemiological and a health economic standpoint is considerable. Although it is a disease of the small airways and the lung, patients with COPD have a significant comorbidity with many symptoms and physical and emotional dysfunctions [6].

Because breathing and swallowing share neuroanatomical substrates and pathways, there must be a precise coordination between them to protect the airway. Despite the essentiality of this coordination for safe and efficient swallowing, there is still a lack of information about how it is impacted by disease in general and by COPD in particular [7]. Swallowing symptoms, or oropharyngeal dysphagia, are clinical symptoms defined as difficulty in effectively moving food and liquid from the mouth to the oesophagus [8]. In this study, dysphagia will refer to oropharyngeal dysphagia unless otherwise indicated. The consequences of dysphagia broadly affect respiratory safety (aspiration) and swallowing efficiency (adequacy of nutrition and hydration by mouth) [9]. The true prevalence is difficult to determine as research typically categorise prevalence according to the concomitant medical disorder (e.g. head/neck cancer, stroke, Parkinson's disease). Determining the true prevalence also depends on the diagnostic instrument used. Despite these limitations it is recognised that dysphagia is a highly prevalent clinical condition affecting $29 \%-64 \%$ of all stroke patients [10] and up to $81 \%$ of people with Parkinson's disease [11]. Some studies have shown that the prevalence of dysphagia among independently living older people is $16 \%$ among the $70-79$-year olds and 33\% among octogenarians [12]. Dysphagia has also been identified as a risk factor for acute exacerbations of COPD [13-15]. As aspiration has been reported in patients with COPD, KOBAYASHI et al. [16] stress the importance of early and accurate identification of those patients with COPD who require a more in-depth assessment of swallowing function.

The diagnosis of dysphagia can be based on functional health status questionnaires, clinical assessments, or instrumental evaluations [17]. The implementation of swallowing screening protocols has reduced pneumonia incidence in patients with stroke [18]. In their systematic review on screening tools for dysphagia, however, ETGES et al. [19] found that the protocols were heterogeneous and developed for patients with different underlying pathologies. A first attempt to develop a screening protocol specific for the COPD population was made by REGAN et al. [20]. In their pilot study in 2017, they investigated the Eating Assessment Tool (EAT)-10 [21] as a protocol to identify adults with stable COPD who aspirate during swallowing.

In a number of studies, the prevalence of swallowing dysfunction has been reported to be higher in patients with stable COPD than in the general population [17, 22-24]. Studies on swallowing dynamics and pulmonary function have concluded that an integrated breathing and swallowing pattern is often suboptimal in patients with COPD, thereby affecting some patients' ability to swallow and to swallow safely [25]. LANGMORE et al. [26] showed that COPD was the strongest independent comorbidity predictor (OR 2.49) for aspiration pneumonia in patients in nursing homes. In a recent systematic review on swallowing function and chronic respiratory disease [17], the outcome data indicate that chronic respiratory diseases increase the prevalence of oropharyngeal dysphagia in patients. Oesophageal dysphagia in the form of gastro-oesophageal reflux has also been reported as more common in patients with COPD [27] and associated with more frequent exacerbations; however, the exact prevalence of either oropharyngeal or oesophageal dysphagia in patients with COPD is not fully understood nor is the disease's impact on swallowing function and the patient's perception of this. Like COPD, the impact of dysphagia is considerable both from an epidemiological and an individual patient perspective; it can lead to aspiration, malnutrition, and a heavy burden on healthcare resources [28].

The aim of this study was to investigate the prevalence of subjective (i.e. self-reported) swallowing symptoms in a large cohort of patients with stable COPD and identify potential related risk factors.

\section{Materials and methods}

Participants

The study data were derived from the Swedish Tools Identifying Exacerbations in COPD-study (TIE-study) which is a prospective, cross-sectional, and multicentre study in primary and secondary care [29]. Patients ( $\mathrm{n}=571 ; 59 \%$ female) from primary and secondary care in three Swedish counties were recruited between September 2014 and September 2016. Inclusion criteria were a spirometry-verified COPD diagnosis, a post-bronchodilator ratio of the forced expiratory volume in $1 \mathrm{~s}\left(\mathrm{FEV}_{1}\right) /$ vital capacity (VC) or forced vital capacity $(\mathrm{FVC})<0.70$; age $\geqslant 40$ years; and the ability to independently fill in the questionnaire and participate in the tests of physical capacity. Patients with severe comorbidities (e.g. dementia, 
metastasised cancer, severe cardiac insufficiency or severe sequelae from stroke) were excluded. None was in an acute phase of the disease. All patients were ambulatory, lived at home (not in a nursing facility) and were taking their full nutrition (liquid and solid) by mouth.

\section{Procedure}

Data were collected in a clinical environment by research nurses and members of the TIE-study group. Spirometries were performed by the trained research nurses. The following investigations were of interest for this substudy: questionnaire, spirometry test and test of physical capacity

\section{Questionnaire}

A questionnaire comprising 61 questions separated by theme was designed to collect information on comorbidity, smoking history, number of COPD exacerbations and exacerbation-related hospitalisations during the previous year. It included the modified Medical Research Council (mMRC), and COPD Assessment Test (CAT), which are two self-rating tools to evaluate the impact of dyspnoea (mMRC) and health-related quality of life (CAT) in patients with COPD [30, 31]. Following the Global Initiative for Chronic Obstructive Lung Disease (GOLD) guidelines [32] we defined patients with COPD as being more symptomatic if they had an mMRC score $\geqslant 2$ or a CAT score $\geqslant 10$.

Swallow-specific data were collected through three questions: (1) I cough when I eat; (2) Food/drink goes down the wrong way when I eat; and (3) Food sticks in my throat when I eat. At the time, there was no validated dysphagia questionnaire available in Sweden. These three questions are some of the strongest clinical signs for identifying oropharyngeal dysphagia [8], they have been used in a previous study on COPD and swallowing [22] and are also used in several validated dysphagia screening protocols [21] Thus, the patients in our study were dichotomised into either a dysphagia or nondysphagia group. Anyone who answered "yes" to any of the questions was accepted into the dysphagia group, since the aim was to study prevalence and not the degree of possible swallowing symptoms.

\section{Spirometry}

Spirometry was performed using either a Spiro Perfect Spirometer (Welch Allyn, Skaneateles Falls, NY, USA) or a Jaeger Master Screen PFT (Erich Jaeger, GmbH, Würtzburg, Germany). A post-bronchodilator ratio (15 min after administration of $400 \mu \mathrm{g}$ salbutamol) of $\mathrm{FEV}_{1} /$ best of $\mathrm{FVC}$ and $\mathrm{VC}<0.7$ was used to define COPD. Swedish reference values [33] were applied for the $\mathrm{FEV}_{1}$

\section{Test of physical capacity}

Physical capacity (endurance and leg muscle strength) was measured by the time to walk 30 metres at maximum speed [34].

\section{Body mass index}

Height and weight were measured at the visit. Body mass index (BMI) was calculated using the formula: weight $(\mathrm{kg}) /$ height $\left(\mathrm{m}^{2}\right)$.

\section{Exacerbation}

An exacerbation was defined as unscheduled visits and/or course of oral steroids and/or a course of antibiotics due to COPD deterioration. Frequent exacerbations were defined as having at least two exacerbations or at least one hospital admittance due to a COPD exacerbation during the year prior to study inclusion.

\section{The GOLD classification}

The goals of COPD assessment are to determine the severity of airflow limitation, its impact on patient's health status and risk of future events such as exacerbations, hospital admissions and death. This is done in two ways: spirometry is performed to assess and classify the airflow limitation (stage 1-4) and secondly an assessment of either dyspnoea using mMRC or health status using CAT together with their history of exacerbations during the last year. This categorises patients into group A, B, C or D [32]. In this study, symptom assessment by CAT was used for risk stratification.

\section{Ethical approval}

The Regional Review Board in Uppsala, Sweden (Dnr 2013/358) approved the study on April 28, 2014. All subjects gave written informed consent.

\section{Statistical analysis}

Descriptive and comparative statistical calculations were made through SPSS statistics, version 24 (IBM, Armonk, NY, USA). Demographic data are reported as means, median, standard deviation and range. 
Group-wise differences for numerical values were tested with an unpaired t-test or Mann-Whitney U-test, whereas categorical values were compared using the Chi-squared test. A p-value $<0.05$ was regarded as significant.

\section{Results}

As can be seen in table 1,33\% $(n=186)$ of the 571 patients reported at least one of the three swallowing symptoms. Participants presented with mild $(n=57)$, moderate $(n=314)$, severe $(n=155)$ and very severe $(\mathrm{n}=45)$ COPD. The most common comorbidity in this study was chronic bronchitis, followed by asthma and heart disease (table 1). No significant relation was found between swallowing symptoms and sex, age, exacerbations or comorbidities $(\mathrm{p}>0.05)$.

\section{TABLE 1 Characteristics of the study population}

\begin{tabular}{|c|c|c|c|}
\hline Characteristics & Total & $\begin{array}{c}\text { Subjective swallowing } \\
\text { symptoms }\end{array}$ & $\begin{array}{l}\text { No swallowing } \\
\text { symptoms }\end{array}$ \\
\hline Subjects & 571 & $186(33 \%)$ & $385(67 \%)$ \\
\hline \multicolumn{4}{|l|}{ Sex } \\
\hline Female & 335 & $112(33 \%)$ & $223(67 \%)$ \\
\hline Male & 236 & $74(31 \%)$ & $162(69 \%)$ \\
\hline Age years & $68.6 \pm 7.6$ & $68.9 \pm 7.8$ & $68.4 \pm 7.6$ \\
\hline \multicolumn{4}{|l|}{ GOLD spirometric stage } \\
\hline Stage $1, \mathrm{FEV}_{1} \geqslant 80 \%$ predicted & 57 & $22(39 \%)$ & $35(61 \%)$ \\
\hline Stage $2, \mathrm{FEV}_{1} 50-<80 \%$ predicted & 314 & $96(31 \%)$ & $218(69 \%)$ \\
\hline Stage $3, \mathrm{FEV}_{1} 30-<50 \%$ predicted & 155 & $56(36 \%)$ & $99(64 \%)$ \\
\hline Stage $4, \mathrm{FEV}_{1}<30 \%$ predicted & 45 & $12(27 \%)$ & $33(73 \%)$ \\
\hline \multicolumn{4}{|l|}{ GOLD 2017} \\
\hline Group A & 188 & $40(21 \%)$ & $148(79 \%)$ \\
\hline Group B & 244 & $98(40 \%)$ & $146(60 \%)$ \\
\hline Group C & 29 & $3(10 \%)$ & $26(90 \%)$ \\
\hline Group D & 110 & $45(41 \%)$ & $65(59 \%)$ \\
\hline \multicolumn{4}{|l|}{ Spirometry } \\
\hline $\mathrm{FEV}_{1} \%$ predicted & $56.6 \pm 17.8$ & $57.0 \pm 18.3$ & $56.3 \pm 17.6$ \\
\hline $\mathrm{FEV}_{1} \mathrm{~L}$ & $2.9 \pm 0.91$ & $2.81 \pm 0.85$ & $3.0 \pm 0.93$ \\
\hline \multicolumn{4}{|l|}{ Smoking status } \\
\hline Current smoker & 165 & $42(26 \%)$ & $123(74 \%)$ \\
\hline Former smoker & 395 & $140(35 \%)$ & $255(65 \%)$ \\
\hline Never-smoker & 9 & $2(22 \%)$ & $7(78 \%)$ \\
\hline \multicolumn{4}{|l|}{ Weight } \\
\hline Stable & 377 & 108 (29\%) & $269(71 \%)$ \\
\hline Weight gain & 111 & $43(39 \%)$ & $68(61 \%)$ \\
\hline Weight loss & 80 & $34(42 \%)$ & $46(58 \%)$ \\
\hline BMI $\mathrm{kg} \cdot \mathrm{m}^{-2}$ & $26.8 \pm 5.1$ & $28.1 \pm 5.6$ & $26.2 \pm 4.6$ \\
\hline Underweight, $\mathrm{BMI}<18.5 \mathrm{~kg} \cdot \mathrm{m}^{-2}$ & 25 & $6(24 \%)$ & $19(76 \%)$ \\
\hline Normal weight, BMI $18.5-24.9 \mathrm{~kg} \cdot \mathrm{m}^{-2}$ & 199 & $54(27 \%)$ & $145(73 \%)$ \\
\hline Overweight, BMI $25-29.9 \mathrm{~kg} \cdot \mathrm{m}^{-2}$ & 207 & $61(29 \%)$ & $146(71 \%)$ \\
\hline Obese, $\mathrm{BMI} \geqslant 30 \mathrm{~kg} \cdot \mathrm{m}^{-2}$ & 137 & $65(47 \%)$ & $72(53 \%)$ \\
\hline \multicolumn{4}{|l|}{ Comorbidities } \\
\hline Stroke/TIA & 50 & $20(40 \%)$ & $30(60 \%)$ \\
\hline Obstructive sleep apnoea & 60 & $27(45 \%)$ & $33(55 \%)$ \\
\hline Rheumatoid arthritis & 65 & $24(37 \%)$ & $41(63 \%)$ \\
\hline Asthma & 189 & $72(38 \%)$ & $117(62 \%)$ \\
\hline Chronic bronchitis & 193 & $80(41 \%)$ & $113(59 \%)$ \\
\hline Heart disease & 114 & $49(43 \%)$ & $65(57 \%)$ \\
\hline \multicolumn{4}{|l|}{ Symptom burden } \\
\hline Dyspnoea, $\mathrm{mMRC}$ score $\geqslant 2$ & 252 & $116(46 \%)$ & $136(54 \%)$ \\
\hline Health-related QoL, CAT score $\geqslant 10$ & 354 & $143(40 \%)$ & $210(60 \%)$ \\
\hline \multicolumn{4}{|l|}{ Exacerbations } \\
\hline$\leqslant 1$ during previous year & 432 & $138(32 \%)$ & $294(68 \%)$ \\
\hline$>1$ or hospital admission & 139 & $48(35 \%)$ & $91(65 \%)$ \\
\hline
\end{tabular}

Data are presented as mean \pm SD unless otherwise stated. GOLD: Global initiative for Obstructive Lung Disease; $\mathrm{FEV}_{1}$ : forced expiratory volume in $1 \mathrm{~s}$; BMI: body mass index;TIA: transient ischaemic attack; mMRC: modified Medical Research Council; QoL: quality of life; CAT: COPD Assessment Test. 


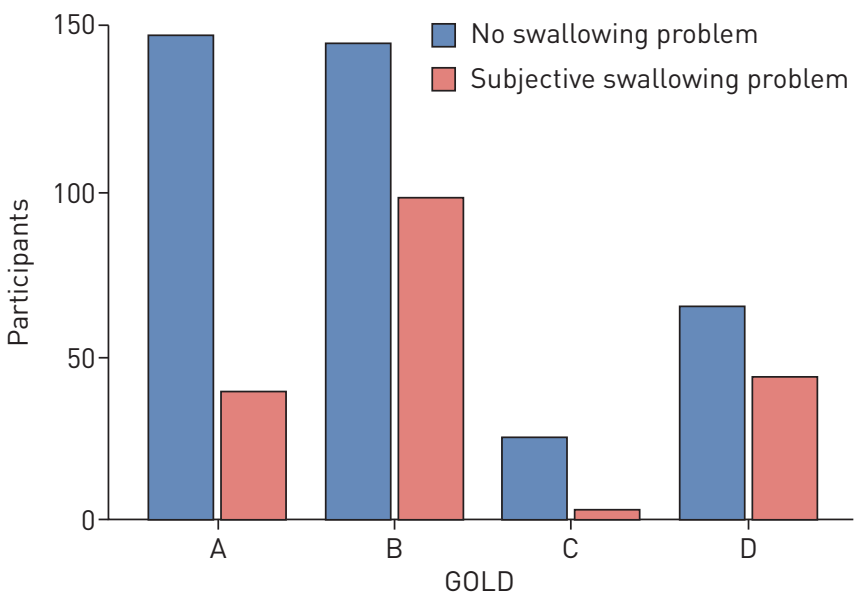

FIGURE 1 Participants with and without subjective swallowing symptoms according to the Global Initiative for Chronic Obstructive Lung Disease (GOLD) 2017 classification.

\section{Subjective swallowing symptoms}

Of the 571 patients, $14 \%$ reported coughing when eating, $22 \%$ reported that "food/drink goes down the wrong way when I eat" and $23 \%$ reported that "food sticks in my throat when I eat". Two of the swallowing symptoms was reported by $53 \%(n=99)$ of the patients and $26 \%(n=49)$ said "yes" to all three. Of these 49 patients who experienced all three swallowing symptoms, $74 \%(n=35)$ were found in the symptomatic dyspnoea group $(\mathrm{mMRC} \geqslant 2), 86 \%(n=42)$ in the group with poorer health-related quality of life $(\mathrm{CAT} \geqslant 10)$ and finally $37 \%(\mathrm{n}=18)$ in the group with frequent exacerbations. Participants with swallowing symptoms were more often in GOLD group B and D than those without swallowing symptoms at $40 \%$ and $41 \%$ compared with $21 \%$ and $10 \%$ respectively, $\mathrm{p}<0.001$ (figure 1 ).

A significant relation was found between swallowing symptoms and dyspnoea, assessed as mMRC $\geqslant 2$ compared with $<2(46 \%, \mathrm{n}=116$ versus $22 \%, \mathrm{n}=70, \mathrm{p}<0.001)$ and health-related quality of life, assessed as CAT $\geqslant 10(40 \%, \mathrm{n}=143$ versus $19 \%, \mathrm{n}=42, \mathrm{p}<0.001)$. Hence the risk of experiencing swallowing symptoms was more than doubled in the groups that were more symptomatic (figure 2).

A similar percentage of participants experienced swallowing symptoms in the different stages of airflow limitation (GOLD 1-4): $39 \%$ in stage 1, 31\% in stage 2, 36\% in stage 3 and $27 \%$ in stage 4 , meaning no significant difference was found $(\mathrm{p}=0.28)$. Subjects with swallowing symptoms needed a longer time to walk $30 \mathrm{~m}$ (median (interquartile range)) than subjects without dysphagia: $19.1 \mathrm{~s}(16.9,23)$ versus $18.4 \mathrm{~s}$ $(16.3,21.5), p=0.02$.

\section{Discussion}

The primary finding of this study was that the prevalence of subjective swallowing symptoms was significantly higher in our COPD population (33\%) than the $2.3-16 \%$ range reported in the general population [35] and the 16\% reported among independently living older persons aged 70-79 years [36]. In their case-control study, CvejIC et al. [13] found that the penetration/aspiration scores were higher in

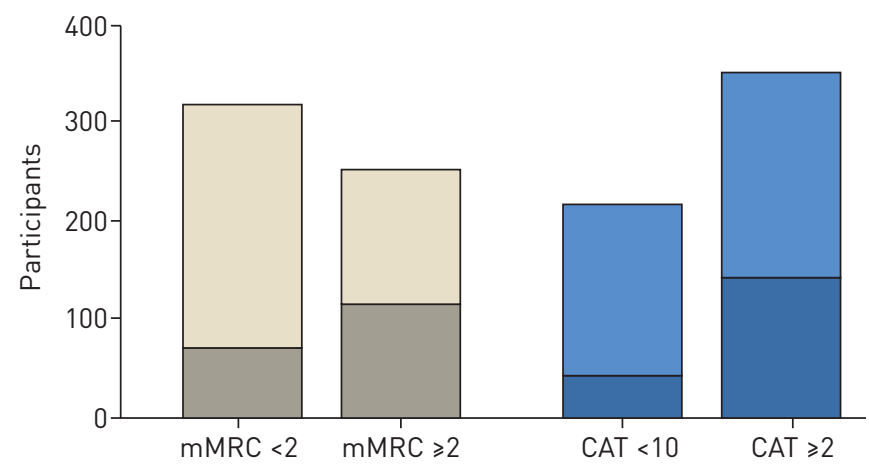

FIGURE 2 Participants with and without subjective swallowing problems according to modified Medical Research Council (mMRC) and COPD Assessment Test (CAT) classification. Darker shading in the bar graph indicates swallowing problems. 
patients with COPD $(3.3 \pm 0.7$ versus $1.6 \pm 0.4)$ than in healthy controls $(\mathrm{p}=0.03)$. Further, penetration/ aspiration was associated with higher respiratory rates $(p=0.01)$ although none of the patients had subjective swallowing complaints. CLAYTON et al. [37, 38] looked at laryngopharyngeal sensitivity in patients with stable COPD and compared the endoscopic evaluation of swallowing with a valid standardised clinical assessment of the same patient and found that patients with no subjective swallowing complaints showed evidence of pharyngeal compensation when compared with a control group. Possible explanations for this unawareness are polypharmacy and the simple fact that coughing is the most recognised sign of aspiration; it might therefore be difficult to distinguish a generic COPD cough from an aspiration cough.

The characteristics of oropharyngeal swallowing symptoms in patients with COPD are not yet defined. The most commonly reported symptom in this study was food lodging in the throat (i.e. bolus retention). This indicates residue in the oropharynx, valleculae, hypopharynx and/or pyriform sinuses. STEIN et al. [24] found that patients with COPD have an increased risk of cricopharyngeal dysfunction, which often results in bolus retention in the pyriform sinuses. Since respiratory and limb muscle fatigue are commonly observed in this patient group [39], it is also possible that the oral and pharyngeal muscles are negatively affected secondary to a general deconditioning due to physical inactivity. This in turn may cause pharyngeal bolus retention.

Another common reason for bolus retention is xerostomia (dry mouth). Chronic xerostomia is a significant burden for many individuals, because it can affect chewing, swallowing, denture-wearing and general wellbeing [40]. Xerostomia is a common problem in patients with COPD due to mouth breathing and the use of inhaled bronchodilators and corticosteroids [20, 41]. In addition, polypharmacy significantly influences patients' saliva.

Overall, $22 \%$ of the patients in our study reported that "food/drink goes down the wrong way when I eat" and $14 \%$ reported coughing when eating. The main sign of aspiration (i.e. food and drink going down the wrong way), is coughing and the main symptom for some patients with COPD is daily coughing [42] which means that it might be difficult for the patient to distinguish a COPD cough from an aspiration cough; hence a chronic cough could potentially mask an aspiration. Because breathing and swallowing share a common pathway, they must be precisely coordinated to protect the airway [7]. Changes in the ventilation pattern can compromise swallowing as CVEJIC et al. [13] also found when they studied the swallowing of 16 patients with COPD using videofluoroscopy while monitoring respiration.

Like dysphagia, dyspnoea is a subjective experience. Our findings of a strong relationship between swallowing symptoms and dyspnoea are perhaps not surprising, since swallowing can unmask or exacerbate dyspnoea. Swallowing requires the usual tidal breathing pattern to be changed to meet certain demands (e.g. higher pressures must be generated and expiration must cease momentarily). The current understanding of dyspnoea in relation to swallowing is that the modification of the breathing pattern necessary for swallowing can create breathing discomfort. In turn breathing discomfort can cause people to alter their swallowing behaviours [43]. A questionnaire-based survey on swallowing-related dyspnoea by HoIт et al. [43], where 133 patients with COPD participated (mean age: 66.5 years) found that $74 \%$ experienced breathing discomfort during eating and drinking. However, no data regarding COPD severity or lung function were provided.

In a previous study, we found a clear negative correlation between lung function and dysphagia burden; the poorer the participants' lung function, the more signs and symptoms of dysphagia [22]. However, in the current study there was no clear association between lung function and subjective swallowing symptoms, as a similar percentage of subjects experienced swallowing symptoms in the different stages of airflow limitation. Possible explanations for this discrepancy may be that in the previous study, the patients were older and more symptomatic, with the majority classified as GOLD stages 3 and $4(74 \%$ versus 35\%), which implies more cases of polypharmacy and comorbidity. Also, the first study screened dysphagia both subjectively and objectively.

In the revised recommendations of GOLD, spirometry has been removed from the risk assessment and is used only to grade the patient's airflow limitation from 1 to 4 . The risk assessment into groups A to D are now based solely on the symptom burden and exacerbation history [32]. Interestingly there was a clear stratification within the group with swallowing symptoms, where groups B and D had a higher prevalence of swallowing symptoms than groups $\mathrm{A}$ or $\mathrm{C}(\mathrm{p}<0.001)$ (figure 1$)$.

Physical activity levels are frequently lower in the COPD population [44]. Walking distance is a predictor of exacerbations in patients with COPD [45]. During pulmonary rehabilitation, a 6-min walk test is typically performed. In this study a $30-\mathrm{m}$ walk test was used and our data suggest a relationship between a longer time to walk $30 \mathrm{~m}$ and subjective swallowing symptoms. 
Dysphagia is also a precipitating factor for malnutrition in COPD [46]. Interestingly, in this study there was no positive correlation between subjective swallowing problems and BMI. In fact, the mean BMI in both groups was in the overweight category. It is well established that patients with COPD have a decreased level of physical capacity, primarily due to breathlessness and therefore easily gain weight. Our data suggest that BMI is not a good variable for identifying dysphagia in patients with COPD.

\section{Strengths and limitations}

The strength of this study was that the study population was sampled from multiple centres, both primary and secondary care facilities. It included patients with different degrees of airflow obstruction and COPD-related symptoms, and the total number of participants included was substantial.

The limitation of this study was that most of the data were gathered through a questionnaire and therefore might be subject to recall bias and not as reliable as data taken only from medical records. The questions regarding swallowing and weight are indeed some of the ones most commonly used worldwide when evaluating a patient with oropharyngeal dysphagia, but our study would have benefited from an accompanying instrumental swallowing examination.

\section{Conclusion}

We conclude that subjective swallowing symptoms are a common problem in patients with COPD and they are more prevalent in symptomatic patients and patients with lower physical capacity. Early detection of swallowing difficulties is important because they can significantly impact a person's quality of life. A high score on the mMRC or CAT and a classification in GOLD groups B and D may be predictive of a swallowing dysfunction and should be addressed by the COPD nurse or physician.

Future research is needed to study the prevalence of oropharyngeal dysphagia in patients with COPD and the relation to A-D grading (disease severity), physical capacity and dyspnoea. These investigations will lead to a better understanding of the risk factors of developing oropharyngeal dysphagia in patients with COPD and how to identify them in clinical practice.

Acknowledgements: The authors are grateful to the research nurses/technicians at the three study sites: Dalarna, Gävle and Uppsala.

Conflict of interest: M. Gonzalez Lindh has nothing to disclose. A. Malinovschi has nothing to disclose. E. Brandén has nothing to disclose. C. Janson has nothing to disclose. B. Ställberg reports personal fees for advisory board meetings, educational activities and lectures from AstraZeneca, Novartis, Boehringer Ingelheim and Meda, for advisory board meetings from GlaxoSmithKline, and for educational activities and lectures from Teva, outside the submitted work. K. Bröms has nothing to disclose. M. Blom Johansson has nothing to disclose. K. Lisspers reports payment for educational activities and lectures from AstraZeneca, Novartis, TEVA, Boehringer Ingelheim and Chiesi and payments for participating on advisory boards for Boehringer Ingelheim and GlaxoSmithKline, outside the submitted work. H. Koyi has nothing to disclose.

Support statement: This study was funded by the Uppsala-Örebro Regional Research Council; Centre for Research and Development, Uppsala University/Region Gävleborg; Centre for Clinical Research, Uppsala University; County Counci Dalarna; The Swedish Heart-Lung Foundation; The Swedish Heart and Lung Association; and The Uppsala County Association against Heart and Lung Diseases. Funding information for this article has been deposited with the Crossref Funder Registry.

\section{References}

1 World Health Organization. www.who.int/respiratory/copd/en/ Date last accessed: October 3, 2018. Date last updated: 2018.

2 Sundh J, Janson C, Johansson G, et al. Characterization of secondary care for COPD in Sweden. Eur Clin Respir J 2017; 4: 1270079.

3 Kruis AL, Ställberg B, Jones RC, et al. Primary care COPD patients compared with large pharmaceutically-sponsored COPD studies: an UNLOCK validation study. PLoS One 2014; 9: e90145.

4 Swedish Heart-Lung Foundation. www.hjart-lungfonden.se/Sjukdomar/Lungsjukdomar/KOL/ Date last accessed: 2018. Date last updated: $3 / 3 / 2017$.

5 Lozano R, Naghavi M, Foreman K, et al. Global and regional mortality from 235 causes of death for 20 age groups in 1990 and 2010: a systematic analysis for the Global Burden of Disease Study 2010. Lancet 2012; 380 2095-2128.

6 Mannino DM, Higuchi K, Yu TC, et al. Economic burden of COPD in the presence of comorbidities. Chest 2015; 148: $138-150$.

7 McFarland DH, Martin-Harris B, Fortin AJ, et al. Respiratory-swallowing coordination in normal subjects: lung volume at swallowing initiation. Respir Physiol Neurobiol 2016; 234: 89-96.

8 Logemann JA. Evaluation and treatment of swallowing disorders. 2nd Edn. Austin, TX, Pro-Ed Inc., 1998.

9 Cichero JA, Altman KW. Definition, prevalence and burden of oropharyngeal dysphagia: a serious problem among older adults worldwide and the impact on prognosis and hospital resources. Nestle Nutr Inst Workshop Ser 2012; 72: 1-11

10 Mann G, Hankey GJ, Cameron D. Swallowing function after stroke: prognosis and prognostic factors at 6 months. Stroke 1999; 30: 744-748. 
11 Wilkins T, Gillies RA, Thomas AM, et al. The prevalence of dysphagia in primary care patients: a HamesNet Research Network study. J Am Board Fam Med 2007; 20: 144-150.

12 Cabre M, Serra-Prat M, Palomera E, et al. Prevalence and prognostic implications of dysphagia in elderly patients with pneumonia. Age Ageing 2010; 39: 39-45.

13 Cvejic L, Harding R, Churchward T, et al. Laryngeal penetration and aspiration in individuals with stable COPD. Respirology 2011; 16: 269-275.

14 Good-Fratturelli MD, Curlee RF, Holle JL. Prevalence and nature of dysphagia in VA patients with COPD referred for videofluoroscopic swallow examination. J Commun Disord 2000; 33: 93-110.

15 Zheng Z, Wu Z, Liu N, et al. Silent aspiration in patients with exacerbation of COPD. Eur Respir J 2016; 48 570-573.

16 Kobayashi S, Kubo H, Yanai M. Impairment of the swallowing reflex in exacerbations of COPD. Thorax 2007; 62 $1017-1017$.

17 Ghannouchi I, Speyer R, Doma K, et al. Swallowing function and chronic respiratory diseases: systematic review. Respir Med 2016; 117: 54-64.

18 Ickenstein GW, Riecker A, Höhlig C, et al. Pneumonia and in-hospital mortality in the context of neurogenic oropharyngeal dysphagia (NOD) in stroke and a new NOD step-wise concept. J Neurol 2010; 257: 1492-1499.

19 Etges CL, Scheeren B, Gomes E, et al. Screening tools for dysphagia: a systematic review. Codas 2014; 26: 343-349.

20 Regan J, Lawson S, De Aguiar V. The Eating Assessment Tool-10 predicts aspiration in adults with stable chronic obstructive pulmonary disease. Dysphagia 2017; 32: 714-720.

21 Belafsky PC, Mouadeb DA, Rees CJ, et al. Validity and reliability of the Eating Assessment Tool (EAT-10). Ann Otol Rhinol Laryngol 2008; 117: 919-924.

22 Gonzalez Lindh M, Blom Johansson M, Jennische M, et al. Prevalence of swallowing dysfunction screened in Swedish cohort of COPD patients. Int J Chron Obstruct Pulmon Dis 2017; 12: 331-337.

23 O'Kane L, Groher M. Oropharyngeal dysphagia in patients with chronic obstructive pulmonary disease: a systematic review. Revista CEFAC 2009; 11: 449-506.

24 Stein M, Williams AJ, Grossman F, et al. Cricopharyngeal dysfunction in chronic obstructive pulmonary disease. Chest 1990; 97: 347-352.

25 Martin-Harris B, Brodsky MB, Michel Y, et al. Breathing and swallowing dynamics across the adult lifespan. Arch Otolaryngol Head Neck Surg 2005; 131: 762-770.

26 Langmore SE, Skarupski KA, Park PS, et al. Predictors of aspiration pneumonia in nursing home residents. Dysphagia 2002; 17: 298-307.

27 Teramoto S. A possible pathological link among swallowing dysfunction, gastro-esophageal reflex and sleep apnea in acute exacerbation in COPD patients. Int J Chron Obstruct Pulmon Dis 2016; 11: 147-150.

28 Dziewas R, Beck AM, Clave P, et al. Recognizing the importance of dysphagia: stumbling blocks and stepping stones in the twenty-first century. Dysphagia 2017; 32: 78-82.

29 Hogman M, Sulku J, Ställberg B, et al. 2017 Global Initiative for Chronic Obstructive Lung Disease reclassifies half of COPD subjects to lower risk group. Int J Chron Obstruct Pulmon Dis 2018; 13: 165-173.

30 Bestall JC, Paul EA, Garrod R, et al. Usefulness of the Medical Research Council (MRC) dyspnoea scale as a measure of disability in patients with chronic obstructive pulmonary disease. Thorax 1999; 54: 581-586.

31 Jones PW, Harding G, Berry P, et al. Development and first validation of the COPD Assessment Test. Eur Respir J 2009; 34: 648-654.

32 Global Initiative for Chronic Obstructive Lung Disease (GOLD) 2019. Global strategy for the diagnosis, management and prevention of COPD, 2019. Available from: https://goldcopd.org/.

33 Berglund E, Birath G, Bjure J, et al. Spirometric studies in normal subjects. I. Forced expirograms in subjects between 7 and 70 years of age. Acta Med Scand 1963; 173: 185-192.

34 Andersson M, Moberg L, Svantesson U, et al. Measuring walking speed in COPD: test-retest reliability of the 30-metre walk test and comparison with the 6-minute walk test. Prim Care Respir J 2011; 20: 434-440.

35 Kertscher B, Speyer R, Fong E, et al. Prevalence of oropharyngeal dysphagia in the Netherlands: a telephone survey. Dysphagia 2015; 30: 114-120.

36 Wirth R, Dziewas R, Beck AM, et al. Oropharyngeal dysphagia in older persons - from pathophysiology to adequate intervention: a review and summary of an international expert meeting. Clin Interv Aging 2016; 11: 189-208.

37 Clayton NA, Carnaby-Mann GD, Peters MJ, et al. The effect of chronic obstructive pulmonary disease on laryngopharyngeal sensitivity. Ear Nose Throat J 2012; 91: 370. 372, 374 passim.

38 Clayton NA, Carnaby GD, Peters MJ, et al. Impaired laryngopharyngeal sensitivity in patients with COPD: the association with swallow function. Int J Speech Lang Pathol 2014; 16: 615-623.

39 Gea J, Pascual S, Casadevall C, et al. Muscle dysfunction in chronic obstructive pulmonary disease: update on causes and biological findings. $J$ Thorac Dis 2015; 7: E418-E438.

40 Villa A, Connell CL, Abati S. Diagnosis and management of xerostomia and hyposalivation. Ther Clin Risk Manag 2015; $11: 45-51$.

41 Theander K, Hasselgren M, Luhr K, et al. Symptoms and impact of symptoms on function and health in patients with chronic obstructive pulmonary disease and chronic heart failure in primary health care. Int J Chron Obstruct Pulmon Dis 2014; 9: 785-794.

42 Miravitlles M, Ribera A. Understanding the impact of symptoms on the burden of COPD. Respir Res 2017; 18 67-67.

43 Hoit JD, Lansing RW, Dean K, et al. Nature and evaluation of dyspnoea in speaking and swallowing. Semin Speech Lang 2011; 32: 5-20.

44 Andersson M, Stridsman C, Rönmark E, et al. Physical activity and fatigue in chronic obstructive pulmonary disease: a population-based study. Respir Med 2015; 109: 1048-1057.

45 Emtner MI, Arnardottir HR, Hallin R, et al. Walking distance is a predictor of exacerbations in patients with chronic obstructive pulmonary disease. Respir Med 2007; 101: 1037-1040.

46 Rofes L, Arreola V, Romea M, et al. Pathophysiology of oropharyngeal dysphagia in the frail elderly. Neurogastroenterol Motil 2010; 22: 851-858. 